\title{
Mental Rotation is Not Easily Cognitively Penetrable
}

\section{Citation}

Borst, Grégoire, Rogier A. Kievit, William L. Thompson, and Stephen M. Kosslyn. Forthcoming. Mental rotation is not easily cognitively penetrable. European Journal of Cognitive Psychology.

\section{Published Version}

http://www.informaworld.com/smpp/title $\sim \mathrm{db}=a$ all content=t713734596

\section{Permanent link}

http://nrs.harvard.edu/urn-3:HUL.InstRepos:4475560

\section{Terms of Use}

This article was downloaded from Harvard University's DASH repository, and is made available under the terms and conditions applicable to Open Access Policy Articles, as set forth at http:// nrs.harvard.edu/urn-3:HUL.InstRepos:dash.current.terms-of-use\#OAP

\section{Share Your Story}

The Harvard community has made this article openly available.

Please share how this access benefits you. Submit a story.

Accessibility 


\title{
Mental Rotation Is Not Easily Cognitively Penetrable
}

\author{
Grégoire Borst*, Rogier A. Kievit*, William L. Thompson*, \\ and Stephen M. Kosslyn* \\ *Department of Psychology, Harvard University, Cambridge, MA, USA
}

Running Head: Mental Rotation and Cognitive Penetration

Corresponding author:

Grégoire Borst

Harvard University

Department of Psychology

836 William James Hall

33 Kirkland Street

Cambridge MA 02138

USA

Tel.: $617-495-3773$

Fax: 617-496-3122

Email: borst@wjh.harvard.edu 


\begin{abstract}
When participants take part in mental imagery experiments, are they using their "tacit knowledge" of perception to mimic what they believe should occur in the corresponding perceptual task? Two experiments were conducted to examine whether such an account can be applied to mental imagery in general. These experiments both examined tasks that required participants to "mentally rotate" stimuli. In Experiment 1, instructions led participants to believe that they could re-orient shapes in one step or avoid re-orienting the shapes altogether. Regardless of instruction type, response times increased linearly with increasing rotation angles. In Experiment 2, participants first observed novel objects rotating at different speeds, and then performed a mental rotation task with those objects. The speed of perceptually demonstrated rotation did not affect the speed of mental rotation. We argue that tacit knowledge cannot explain mental imagery results in general, and that in particular the mental rotation effect reflects the nature of the underlying internal representation and processes that transform it, rather than participants' pre-existing knowledge.
\end{abstract}

Keywords: visual mental imagery, mental rotation, tacit knowledge, cognitive penetration. 
An enormous amount of research followed Shepard and Metzler's (1971) seminal article on mental rotation. In Shepard and Metzler's paradigm, participants are asked to view three-dimensional objects that have several arms, each consisting of small cubes. The task is to decide whether two such objects have the same shape, regardless of a difference in orientation. The key finding was that response times (RTs) increased linearly with increasing angular disparity between the two objects. Shepard and Metzler interpreted this finding as showing that people mentally rotate one of the objects to align it to the orientation of the other object. Subsequent studies showed that the mental rotation effect was not limited to abstract geometric shapes. The linear increase in RTs has been found in studies using alphanumeric stimuli (e.g., Cooper \& Shepard, 1973, Koriat \& Norman, 1985), two-dimensional line drawings of letter-like asymmetrical characters (e.g., Tarr \& Pinker, 1989), and pictures of common objects (e.g., Jolicoeur, 1985).

Many researchers have interpreted the mental rotation effect (i.e., the linear increase in RTs with an increasing angle between objects) as showing that an internal representation is structurally analogous to a visual percept, and this representation is manipulated in a way that emulates the corresponding physical transformation. This interpretation in turn suggests that one purpose of imagery is to simulate the world to extract previously unnoticed information, for example 
that two shapes in different orientations are in fact identical (Shepard \& Cooper, 1982; see also Kosslyn, Thompson, \& Ganis; Thompson, Kosslyn, Hoffman \& van der Kooij, 2008).

However, Pylyshyn (1981, 2002, 2007) has argued that many classic mental imagery findings do not reflect properties of the underlying representation. Instead, he claims that the results occur because participants use their beliefs (based on experience) to predict or mimic what would happen in a particular situation. According to Pylyshyn, findings in mental imagery studies often reveal nothing about the properties of the underlying representations because they are a byproduct of participants' tacit knowledge. A corollary of this position is that unless proven otherwise, mental imagery data should be interpreted as revealing tacit knowledge, which in turn alters processing (and hence such processing is said to be "cognitively penetrable. Thus, in order to conclude that a given result reflects characteristics of the manner in which an internal representation has been processed, one must demonstrate that different beliefs do not yield different results. Moreover, as Pylyshyn (2007) has emphasized, one should not attempt to introduce such beliefs by inducing "strange expectancies by telling unrealistic stories about object movements" (p. 134) to the participants.

To be clear: we do not claim that Pylyshyn has attributed all mental rotation effects to tacit knowledge. In fact, he has shown that mental rotation slopes can be influenced by task and stimulus parameters (such as practice, 
complexity and "goodness of fit" of the original stimuli with the comparison stimuli; Pylyshyn, 1979), and interpreted this finding as demonstrating that mental rotation is accomplished in a piecemeal, analytic fashion, rather than as the rotation of an analog representation. But our goal is not to test specifically Pylyshyn's views of mental imagery or mental rotation. The general issue of cognitive penetrability -- that is, whether one's beliefs about mental events can influence how one performs a task -- is important in its own right. If one's beliefs about a task can influence how it is performed, data from the task need not reflect characteristics of the underlying representations or processes. If, however, one's beliefs (implicit or explicit) do not affect task performance (e.g., by altering the linear increase in time to rotate farther distances during mental rotation), then this is good evidence that performance characteristics do reflect characteristics of the underlying representations and processes.

In the experiments reported here, we investigated whether tacit knowledge can account for the linear relationship between RTs and angular disparity in mental rotation tasks. We made two strong predictions: First, if the linear relationship between the response time and angular disparity is a consequence of the nature of the underlying representation and process, then participants' expectancies and knowledge about the experimental situation should not alter this relationship. Second, if mental rotation results reflect the inherent nature of the representation and process, then participants' expectancies and knowledge should 
not alter the speed at which they mentally rotate. These predicted null effects should persist in the face of the usual positive effects, specifically the tight relationship between response time and angular distance of rotation.

\section{Experiment 1}

The goal of Experiment 1 was to compare the effect of three types of instructions on two classic mental rotation tasks. One of the tasks required the participants to compare three-dimensional objects adapted from Shepard and Metzler's paradigm (1971, hereafter referred to as the "SM task"), and one of the tasks required them to classify misoriented alphanumeric characters adapted from Cooper and Shepard's paradigm (1973, hereafter referred to as the "CS task"). Participants in different conditions received different sets of realistic instructions, a method proposed by Pylyshyn (1981) as a way to test the tacit knowledge account of mental imagery processes. The different instructions were partly based upon strategies identified by Geiser, Lehmann, and Eid (2006) in a sample of 1,695 participants who performed the 24 items of the Mental Rotation Test (MRT, Vandenberg \& Kuse, 1978).

Specifically, we asked participants to use one of three different strategies to perform the tasks: a mental rotation strategy, a leap strategy, or an analytic strategy. In the mental rotation condition, we asked participants to mentally rotate the object on the right side of the screen from its original orientation to the same 
orientation as the object on the left (SM task), or to rotate the letter or digit to its upright orientation (CS task), and then to make the appropriate judgment. In the leap condition, we asked participants to imagine the object jumping, quickly and in a single leap, from its original orientation to the orientation of the object on the left (SM task), or to the upright orientation (CS task), without visualizing the intermediate steps (as if the transformation were instantaneous). In the analytic condition, we asked participants to compare the segments of the object on the right with the segments of the object on the left (SM task), or to compare the segments of the characters with those in a normal character (CS task), to decide whether they fit together in the same way. The analytic condition we designed is distinct from the strategy identified by Just and Carpenter (1976), where people rotate one segment at a time and then compare how the segments fit together. The analytic condition we used resembles a strategy proposed by Cooper (1976), where participants use reasoning to evaluate the stimuli. Moreover, in the leap and the analytic conditions, participants were explicitly instructed not to mentally rotate the objects or the characters.

If the usual instructions lead participants to use their beliefs of how things unfold in the real world to mimic what would happen when they imagine these situations, then we would not expect a linear relationship between the RTs and the angular disparity of the stimuli in both the leap and the analytical conditions. In fact, if the leap and analytic strategies are effective for performing the tasks, the 
angular disparity of the objects should have no effect on the time required to perform the judgment. On the other hand, if mental rotation occurs because of the structure of the internal representations and the manner in which they are processed in order to perform the tasks, then RTs should increase as more rotation is required regardless of type of instructions. In addition, the rotation rate (as revealed by the slopes of the best-fitting lines), the efficiency of the cognitive process (as revealed by the error rates), and the height of the intercepts should be similar in the three groups of participants if similar cognitive processes and representations are being used.

\section{Method}

\section{Participants}

Seventy-two volunteers from Harvard University and the local community participated in this study ( 44 females and 28 males, mean age 22 years and 4 months). Participants were randomly assigned to one of the three groups (mental rotation, leap, analytic); 68 participants were right- handed, 4 left-handed, and all reported normal or corrected-to-normal vision. Data from 3 participants were not analyzed because they failed to reach a performance threshold on at least one of the tasks. All the participants provided written consent and received either $\$ 10$ or class credit for their participation. Participants were tested in accordance with national and international norms governing the use of human research 
participants. The study was approved by the Harvard University Faculty of Arts and Sciences Committee on the Use of Human Subjects.

\section{Materials}

For the SM task, we created 12 three-dimensional arm-like objects formed by small cubes. All objects consisted of four arms created by connecting 7 to 11 white cubes "(For details on how the stimuli were created, see Wright et al., 2008.)"

As shown in Figure 1, we created a stimulus by placing two objects on a $28 \mathrm{~cm} \mathrm{x}$ $14.5 \mathrm{~cm}$ black rectangle $\left(26.2^{\circ} \times 13.7^{\circ}\right.$ visual angle). The centroid (i.e., the point that was on average closest to all other points of an object) of each object was placed $6 \mathrm{~cm}\left(5.7^{\circ}\right.$ visual angle) to the right or to the left of the center of the black rectangle. Each stimulus was displayed in the center of the screen. The object on the right was either the same as the object on the left or it was a mirror reflection (through the y-axis). The object on the right could be either in the same orientation as the one on the left or rotated by 50,100 , or 150 degrees around the y-axis (see Figure 1).

Insert Figure 1 about here 
For the CS task, we designed 12 non-symmetrical alphanumeric characters: three uppercase letters $(\mathrm{G}, \mathrm{J}, \mathrm{R})$, three numbers $(2,5,7)$, and six mirror-images of those characters. All were shown in black using a 72-point Arial font (extending over $7.62 \mathrm{~cm}$, subtending $7.3^{\circ}$ visual angle) and placed in the center of a $17.5 \mathrm{~cm}\left(16.6^{\circ}\right.$ visual angle) diameter circle (see Figure 2). All 12 characters could be presented at one of six possible orientations (from 0 to 300 degrees with 60 degree intervals).

We presented the stimuli on a 17 in iMac G5 with resolution of $1,680 \mathrm{x}$ 1050 pixels and a refresh rate of $75 \mathrm{~Hz}$. The tasks were implemented using PsyScope software running in Macintosh OS X.

Insert Figure 2 about here

\section{Procedure}

The participants were tested individually, sitting approximately $60 \mathrm{~cm}$ from a computer screen. We assigned each participant to one of the three conditions, and all participants performed both the SM and the CS tasks. The order of the two tasks was fully counterbalanced over participants in each condition. Each group received a different set of instructions (i.e., mental rotation, leap, or analytic). In each task, we first showed the participants written 
instructions (see Appendix for the text of the instructions). Following this, we presented an example of a trial and the way the instructions should be followed. Finally, we asked participants to paraphrase the instructions to ensure that they understood how they should perform the tasks. We administered this procedure at the beginning of each of the two tasks.

Each trial started with a $250 \mathrm{~ms}$ blank screen, after which one of the stimuli was presented until participants responded by pressing one of two buttons within a time limit of $7500 \mathrm{~ms}$. Participants were instructed to make their judgments as quickly and accurately as possible, using the specific instructions they were given at the beginning of the task. In the SM task, participants were to decide whether or not the two objects were identical, and in the CS task they were to decide whether the character was in its standard form or mirror-reversed. Participants used their dominant hand to respond, pressing the " $b$ " key if they decided that the objects in a pair had the same shape (SM task) or if the character was in its standard form (CS task); if they decided that the objects were different or the character was mirror reversed, they pressed the " $n$ " key. The onset of a stimulus started a timer, which was stopped when either of the two response keys was pressed.

In each of the tasks, participants performed two blocks of 48 trials, for a total of 96 trials. In each block, the six possible orientations in the CS task (four orientations in the SM task) occurred equally often. On half of the trials the 
objects in a pair had the same shape (SM task) or the character was in its standard form (CS task). The order of the trials was random, except that no more than three same or different (standard or mirror-image) trials occurred consecutively. In each task, before the first experimental trial, participants performed 12 practice trials where the computer provided feedback on their answer.

At the end of the experiment, the participants completed the Edinburgh Handedness Inventory (Oldfield, 1971), the Object Spatial Imagery Questionnaire (OSIQ, Blajenkova, Kozhevnikov, \& Motes, 2006), the Subjective Use of Imagery Scale (SUIS, Kosslyn laboratory, in development), the Vividness of Visual Imagery Questionnaire (VVIQ, Marks, 1973), and a debriefing questionnaire to ensure that they did not infer the purpose of the experiment and that they followed the instructions.

\section{Results}

All analyses of RTs included only data from trials on which participants provided the correct answer. As is standard practice in studies of mental rotation, we only analyzed data from trials in which the two shapes matched (SM task) or where the character was in its normal form (CS task). This convention has been adopted because the angle through which the "different" shapes must be rotated to attempt to achieve congruence in the SM task is not strictly defined; the same logic applied for the character stimuli in the CS task. Outliers were defined as 
RTs greater than 2 standard deviations from the mean of that angle for that participant. Outliers occurred on $1.8 \%$ of the trials. After removing outliers, for each participant, the average RT for each angle of rotation in each of the two tasks was computed.

As a first step, we analyzed the data from each of the three groups in each task separately to determine (1) whether RTs differed significantly for the different angles, and (2) whether RTs increased linearly with increasing angles in all groups. Following this, we conducted a three-way analysis of variance (ANOVA) to investigate whether there was an interaction between the type of instruction, the type of task, and angular disparity. Finally, we compared the error rates (ERs), the steepness of the slopes of the best-fitting lines, and the heights of the intercepts of the best-fitting lines (i.e. the RTs for the 0 degree orientation) for all three groups in each of the two mental rotation tasks. In addition, for each of the analyses, we report the effect size of the ANOVA (partial eta squared) or of the difference of the means (Cohen's $d$ ).

Preliminary analyses revealed no effect of gender or of task order on any of the dependent variables. Thus, we pooled the data over these variables. Moreover, scores on the three subscales (verbal, spatial and object) of the OSIQ, scores on the VVIQ, and scores on the SUIS did not differ significantly among the three instruction-set groups. Finally, the analysis of the debriefing 
questionnaire revealed that all participants whose data were included in the analyses reported carefully following the instructions.

\section{Effects of angle}

SM task. A one-way repeated measures ANOVA confirmed that RTs varied for different angular disparities between the shapes in all three groups, with $\mathrm{F}(3,69)=65.92, \mathrm{p}<.0001, \eta \mathrm{p}^{2}=.74$, for the mental rotation group, $\mathrm{F}(3,69)=$ 63.32, $\mathrm{p}<.0001, \eta p^{2}=.74$, for the leap group, and $\mathrm{F}(3,69)=74.84, \mathrm{p}<.0001$, $\eta \mathrm{p}^{2}=.77$ for the analytic group. As shown in Figure 3, RTs increased linearly with increasing angular disparity between the two objects, in the mental rotation group $\left[F(1,23)=187.49, \mathrm{p}<.0001, \eta p^{2}=.73\right]$, in the leap group $[F(1,23)=$ 182.27, $\left.\mathrm{p}<.0001, \eta \mathrm{p}^{2}=.73\right]$, and in the analytic group $[\mathrm{F}(1,23)=215.87, \mathrm{p}<$ $\left..0001, \eta p^{2}=.76\right]$. The quadratic trend in the angular disparity effect was also significant, with respectively, $F(1,23)=9.37, \mathrm{p}<.01, \eta \mathrm{p}^{2}=.12$, for the mental rotation group; $\mathrm{F}(1,23)=7.79, \mathrm{p}<.025, \eta \mathrm{p}^{2}=.10$, for the leap group; $\mathrm{F}(1,23)=$ $8.69, \mathrm{p}<.01, \eta \mathrm{p}^{2}=.11$, for the analytic group. However, the linear trend in the effect of angular disparity between objects accounted for between $94.8 \%$ and $96.1 \%$ of the variance due to orientation in the three groups.

In addition, for each of the three groups, we computed the best-fitting linear functions calculated by the method of least squares. RTs and angular disparity between objects were highly correlated (with Bravais-Pearson $r$ s ranging 
from .97 to $.98, \mathrm{p}<.05$ in all groups). These results replicate earlier findings (Shepard \& Metzler, 1971) and suggest that participants mentally rotated the object on the right to achieve congruence before judging whether the two objects had the same shape, regardless of the type of instructions participants were given. In fact the coefficient of correlations between RTs and angular disparity did not differ among the three groups, $\mathrm{p}>.25$ in all cases.

Insert Figure 3 about here

CS task. Again, one-way repeated measures ANOVAs revealed a significant effect of the magnitude of angle on the time to decide whether the character was presented in its standard form. This finding characterized all three groups of participants: in the mental rotation group, $\mathrm{F}(3,69)=50.75, \mathrm{p}<.0001$, $\eta p^{2}=.69$; in the leap group, $\mathrm{F}(3,69)=48.64, \mathrm{p}<.0001, \eta \mathrm{p}^{2}=.68$; and in the analytic group, $\mathrm{F}(3,69)=58.14, \mathrm{p}<.0001, \eta \mathrm{p}^{2}=.72$. Moreover, RTs increased linearly with increasing rotation of the character from its upright position for each group, with $\mathrm{F}(1,23)=137.18, \mathrm{p}<.0001, \eta \mathrm{p}^{2}=.67$, for the mental rotation group; $F(1,23)=130.6, p<.0001, \eta p^{2}=.65$, for the leap group; $F(1,23)=157.39, p<$ $.0001, \eta \mathrm{p}^{2}=.70$, for the analytic group. Although the trend analysis revealed a significant quadratic component in the mental rotation group $[F(1,23)=12.88, p$ 
$\left.<.005, \eta \mathrm{p}^{2}=.16\right]$, in the leap group $\left[\mathrm{F}(1,23)=13.79, \mathrm{p}<.005, \eta \mathrm{p}^{2}=.17\right]$, and in the analytic group $\left[F(1,23)=16.73, p<.001, \eta p^{2}=.20\right]$, the proportion of variance accounted for by the linear trend ranged from $89.5 \%$ to $90.1 \%$ in the three groups of participants.

In addition, as shown in Figure 4, RTs were highly correlated with the angular distance of rotation for all three groups (Bravais-Pearson $r$ s ranging from .95 to $.96, \mathrm{p}<.05)$. The results demonstrated that participants mentally rotated the character from the orientation in which it was presented to its upright orientation, as reported by Cooper and Shepard (1973). Furthermore, the type of instructions given to the participants did not affect the relationship between the RTs and the degree of rotation of the characters, $\mathrm{p}>.25$.

Finally, a three-way ANOVA (Instructions x Task x Angle) provided no evidence that the effect of the angular disparity varied for the two tasks or the type of instructions, $\mathrm{F}(6,207)<1$, NS, $\eta p^{2}<.01$. In each of the two tasks, twoway ANOVAs confirmed that the magnitude of the angular rotation was not affected by the type of instructions, with all $\mathrm{F}$ values $<1$, NS, $\eta \mathrm{p}^{2}<.01$.

\section{Insert Figure 4 about here}

ER, slopes and intercepts 
$E R s$. ERs were consistent for the three instructions and the type of task, $\mathrm{F}$ $<1$, NS, $\eta \mathrm{p}^{2}<.01$, for the interaction between the two variables. In addition, participants made comparable numbers of errors in all three groups in each of the two tasks. In the SM task, the average ERs did not differ between the mental rotation group $(\mathrm{M}=11.8 \%)$, the leap group $(\mathrm{M}=12.5 \%)$, and the analytic group $(\mathrm{M}=13.1 \%)$, all ts $<1, \mathrm{NS}, d s<.37$ (see Table 1). Similarly, in the CS task, participants made comparable numbers of errors in the mental rotation group (M $=4.8 \%)$, the leap group $(\mathrm{M}=5.2 \%)$, and the analytic group $(\mathrm{M}=4.4 \%)$, ts $<1$, $\mathrm{NS}, d s<.62$. Finally, participants made more errors in the SM task $(\mathrm{M}=12.5 \%)$ than in the CS task $(M=4.8 \%), t(71)=6.68, p<.0001, d=1.01$, which is consistent with differences in accuracies previously documented between twodimensional and three-dimensional object rotation (e.g., Carpenter \& Just, 1978; Shepard \& Metzler, 1988).

Insert Table 1 about here

Slopes. For each participant, we computed the slope of the best fitting lines and then averaged the individual slopes over the participants in each group (see Table 1). A two-way ANOVA (Task x Instructions) on the average slopes of the best-fitting lines revealed no interaction between these two variables, $\mathrm{F}<1$, 
NS, $\eta p^{2}=.001$. Two one-way ANOVAs (one for each of the tasks) revealed no effect of the type of instructions on the average speed with which participants mentally rotated the stimuli (reflected by the steepness of the slopes of the best fitting lines), with $\mathrm{F}<1, \mathrm{NS}, \eta \mathrm{p}^{2}=.009$, in the SM task and $\mathrm{F}(2,69)=1.25, \mathrm{p}>$ $.25, \eta p^{2}=.04$, in the CS task. In addition, in the SM task, the steepness of the slopes was comparable in the mental rotation group $(\mathrm{M}=12.4 \mathrm{~ms} /$ degree $)$, the leap group $(\mathrm{M}=11.5 \mathrm{~ms} /$ degree $)$ and the analytic group $(\mathrm{M}=12.8 \mathrm{~ms} /$ degree $)$, all ts $<1$, NS, $d s<.23$. The same was true in the CS task; for the mental rotation group $(\mathrm{M}=4.1 \mathrm{~ms} /$ degree $)$ versus the leap group $(\mathrm{M}=3.3 \mathrm{~ms} /$ degree $), \mathrm{t}(46)=$ $1.31, \mathrm{p}>.15, d=.38$; for the mental rotation group versus the analytic group (M $=4.2 \mathrm{~ms} /$ degree$), \mathrm{t}<1, d=.03$; and for the leap group versus the analytic group, $\mathrm{t}(46)=1.47, \mathrm{p}>.1, d=.42$.

On their face, the results of these analyses appear to support the null hypothesis, which posits no difference between the average slopes between the groups. However, as always, it is difficult to affirm that no difference exists: we risk a type-II error if we lack statistical power. Thus, we conducted a post-hoc power analysis using G*Power (Faul, Erdfelder, Lang, \& Buchner, 2007) to determine the probability of a type-II error given our sample size $(\mathrm{n}=72)$ in each task. If we assume that tacit knowledge is at work, the crucial prediction is that the slopes in the two non-mental rotation groups (i.e., leap and analytic) should be flat (or at least not significantly different from $0 \mathrm{~ms} /$ degree), and should be 
significantly lower than in the mental rotation group. In order to estimate the effect size for the power analysis, we began by computing both the $99 \%$ confidence intervals (CI) in the leap and the analytic groups for an average slope of $0 \mathrm{~ms} /$ degree and the standard deviations based on the largest value observed in the three groups $(S D=5.73 ; \mathrm{CI}=-3.1 \mathrm{~ms} /$ degree to $3.1 \mathrm{~ms} /$ degree in the $\mathrm{SM}$ task and $S D=2.33 ; \mathrm{CI}=-1.2 \mathrm{~ms} /$ degree to $1.2 \mathrm{~ms} /$ degree in the $\mathrm{CS}$ task); we computed the same for the mental rotation group $(\mathrm{CI}=9.5 \mathrm{~ms} /$ degree to 15.5 $\mathrm{ms} /$ degree in the $\mathrm{SM}$ task and $\mathrm{CI}=2.9 \mathrm{~ms} /$ degree to $5.3 \mathrm{~ms} /$ degree in the $\mathrm{CS}$ task). Then, based on those average slopes, we estimated the effect size $(d=1.11$ in the SM task and $d=.78$ in the CS task). For a criterion of significance set at .05 with a two-tailed t-test, the probability of a type-II error was low in the SM task $(\beta=3.6 \%)$ but relatively high in the CS task $(\beta=24.6 \%)$.

Finally, consistent with previous findings (e.g. Carpenter \& Just, 1978; Shepard \& Metzler, 1988), participants mentally rotated the characters $(\mathrm{M}=3.9$ $\mathrm{ms} /$ degrees $)$ faster than the three-dimensional objects $(\mathrm{M}=12.2 \mathrm{~ms} /$ degrees $)$, $\mathrm{t}(71)=13.34, \mathrm{p}<.0001, d=1.97$

Intercepts. We used the mean RTs in the 0 degree orientation in both the SM and the CS tasks as the height of the intercept (see Table 1) in the following analysis. The effect of the type of instructions and the types of tasks did not interact with the height of the intercepts, as revealed by a two-way ANOVA (Task $\mathrm{x}$ Instruction), $\mathrm{F}(2,69)=1.52, \mathrm{p}>.2, \eta \mathrm{p}^{2}=.04$. One-way ANOVAs 
revealed no effect of the type of instructions on the height of the intercepts in the

SM task, $\mathrm{F}<1, \eta \mathrm{p}^{2}=.02$, or in the CS task, $\mathrm{F}(2,69)=2.06, \mathrm{p}>.1, \eta \mathrm{p}^{2}=.06$. In the SM task, the height of the intercepts did not differ between the mental rotation group $(M=1516 \mathrm{~ms})$, the leap group $(\mathrm{M}=1407 \mathrm{~ms})$, and the analytic group $(\mathrm{M}=$ $1395 \mathrm{~ms}$ ), all $t$ values $<1, d s<.24$. Similarly, in the CS task, the mental rotation group $(\mathrm{M}=822 \mathrm{~ms})$ did not differ from the leap group $(\mathrm{M}=814 \mathrm{~ms}), \mathrm{t}<1, d=$ .05 , nor from the analytic group $(\mathrm{M}=924 \mathrm{~ms}), \mathrm{t}(46)=1.64, \mathrm{p}>.1, d=.47$, and the leap group did not differ from the analytic group, $\mathrm{t}(46)=1.63, \mathrm{p}>.1, d=.48$. As reported in the analysis of the ERs and the slopes, the height of the intercept was higher in the SM task $(\mathrm{M}=1439 \mathrm{~ms})$ than in the CS task $(853 \mathrm{~ms}), \mathrm{t}(71)=$ $11.14, \mathrm{p}<.0001, d=1.62$, in line with findings from Carpenter and Just (1978), and Shepard and Metzler (1988).

\section{Discussion}

When participants were required to judge whether two three-dimensional objects were identical (the SM task), they took more time as the angular disparity increased between the two objects. This finding was not affected in any respect by the instructions. Critically, the rate with which participants mentally rotated the objects (as revealed by the steepness of the slopes of the best fitting lines) was not affected by the type of instructions they received. Indeed, the three types of instructions led to comparable slopes, intercepts, and error rates. 
These results suggest that, independently of the type of instructions provided, participants imagined one of the objects rotating into congruence with the other in order to judge whether the two objects had the same shape. Given that participants in the leap group and in the analytic group were not instructed to visualize the object moving along a trajectory (in fact, in the leap condition they were specifically instructed to not imagine the intermediate positions), the strong linear increase in RTs with greater angular disparity observed in all three groups contradicts explanations based on the tacit knowledge account of the mental rotation effect. In addition, it is important to note that the finding of a significant linear relationship between RTs and the angular disparity in the leap and analytic groups, where we would not expect this pattern under the tacit knowledge account of mental rotation, rules out any concerns that our analysis lacked statistical power.

One might be concerned that the lack of an effect of the type of instructions might be restricted to one type of task, one type of stimulus, or one type of rotation axis. We included two tasks in order to assuage such concerns. In fact, in our study, participants mentally rotated unfamiliar three-dimensional objects at a slower rate and less efficiently than well known two-dimensional stimuli, which replicates previous findings (e.g., Carpenter \& Just, 1978; Shepard \& Metzler, 1988). By showing that participants' assumptions about the task do not account for the mental rotation effect in two mental rotation tasks that differ in 
speed and processing efficiency, which affect the characteristics of the cognitive process, we provide evidence that our findings are generalizable.

In addition, one could argue that our manipulation of the instructions was not strong enough to alter the relationship between RTs and angular disparities. However, Pylyshyn (1981) and Kosslyn, Ball and Reiser (Experiment 3, 1978) demonstrated that the mental image scanning effect (i.e., linear increase in RTs with increasing distance scanned) was not observed when participants were not given instructions to scan. Thus, we are confident that the manipulation of the instructions should have produced different RT functions if tacit knowledge accounts for the mental rotation effect.

Finally, in the CS task, although not statistically significant, we did find a trend for smaller effects in the leap group, as predicted by the tacit knowledge account -- and the difference in rotation rate appeared to be greater than "small," according to Cohen's (1988) $d$ benchmarks of effect size. The effect size may suggest that with a larger sample we might have found that participants were faster in the leap group than in the two other groups. In fact, the probability of committing a type II-error on the slopes in this task was relatively large. However, we did observe a linear increase of the RTs with increasing rotation in the leap group (as in the other two groups), which suggests that participants were mentally rotating the stimuli. On this basis, we argue that the trend reflects a tendency toward a speed/accuracy trade-off, given that participants in the leap 
group tended to make more errors than in the two other groups. There is no evidence that cognitive processes distinct from mental rotation were at play in the leap group.

To examine more closely the cognitive penetrability of mental rotation, we conducted an additional experiment. Experiment 2 is intended to manipulate directly the tacit knowledge of the participants about the speed of the rotation. By using a mixed design in this experiment, we also increased statistical power.

\section{Experiment 2}

In Experiment 1, we used instructions in an attempt to discover whether mental rotation is cognitively penetrable. However, Pylyshyn (1981, 2003, 2007) has emphasized the effects of tacit knowledge per se in affecting performance. Thus, in this experiment we manipulated the participants' tacit knowledge of how quickly novel shapes rotate. By using novel objects, we attempt to create tacit knowledge of how these objects behave. After asking the participants to observe a movie of these novel shapes rotating (either relatively quickly or relatively slowly, for different participants), we then investigated whether differences in such experience affect subsequent mental rotation of those objects.

In this experiment, participants mentally rotated the three-dimensional objects (see Figure 1) in the same way as in the mental rotation condition of the SM task in Experiment 1. They performed this task twice, once before (as a 
baseline) and once after a perceptual rotation task. In the perceptual rotation task, before each trial the participants saw a series of single objects completing a 360degree rotation either clockwise or counterclockwise around the y-axis. Prior to showing participants the rotating objects, we explicitly instructed them to pay attention to the rotation rate of the objects because they would later need to mimic the rotation speed in a subsequent mental rotation task. To ensure that participants actually watched the objects rotating, after each trial they were to indicate whether the rotation had been clockwise or counterclockwise. For one group of participants, the objects rotated approximately twice as quickly as the average rotation speed found in the SM task of Experiment 1. For the other group, the objects rotated approximately twice as slowly as the average rotation speed found in the SM task of Experiment 1.

We reasoned that if participants use tacit knowledge of the way things unfold in the real world to mimic what would happen when the situation is imagined, then the speed with which participants mentally rotate the objects (as revealed by the steepness of the slopes of the best-fitting lines) should be affected by the rotation rate of objects displayed visually. Thus, participants who observed these novel objects rotating quickly prior to the mental rotation task should mentally rotate these objects faster than participants who observed the objects rotating slowly prior to the mental rotation task. Conversely, if mental rotation speed is a consequence of the way representations are processed, then we do not 
expect an effect of the rotation rate of the visually presented objects on subsequent performance of the task.

However, we did expect participants to be generally faster in the second set of mental rotation trials than in the first set of trials because of practice effects. Thus, the information-processing view does not simply predict null effects: Rather, we should find the heights of the intercepts of the best-fitting lines to be reduced, but the slopes of the best-fitting lines to remain the same.

\section{Method}

\section{Participants}

We tested 24 volunteers (14 females and 10 males) from Harvard University and the local community who did not participate in the previous experiment. One participant was left-handed and all others were right -handed. The average age of the participants was 22 years and 11 months. Participants received either $\$ 10$ or class credit for their participation, and reported normal or corrected-to-normal vision. Participants were randomly assigned to each of two groups (fast visual rotation task or slow visual rotation task). All the participants provided written consent and were tested in accordance with national and international norms governing the use of human research participants. The study was approved by the Harvard University Faculty of Arts and Sciences Committee on the Use of Human Subjects. 


\section{Materials}

For the mental rotation tasks, we created 24 three-dimensional arm-like objects formed by small cubes, respecting the same characteristics as those designed for the SM task in Experiment 1 (with same number of arms and white cubes, same size, same position on the computer screen, same rotation of the object on the right around the y-axis). In addition, for the perceptual task, we designed 12 additional three-dimensional objects, respecting the same constraints as the 24 stimuli designed for the mental rotation task. For each of these stimuli, we created two animations, which varied in speed (as described below) that rotated 360 degrees on a black background. Animations were 640 x 480 .avi movies (30 frames per second).

\section{Procedure}

We tested the participants individually, with each sitting approximately 60 $\mathrm{cm}$ from a computer screen. We divided the participants into groups of 12 participants, and assigned each group to one of the two conditions (fast visual rotation task or slow visual rotation task). In each group, participants performed a mental rotation task (hereafter referred as MR1), then a visual rotation task (hereafter referred as VR), and finally a second mental rotation task (hereafter referred as MR2). We explicitly asked participants to pay attention to the speed with which the visually presented objects rotated. We told them that they would 
soon be asked to imagine similar objects rotating at the same speed in a subsequent task (i.e., the MR2 task). Before starting the MR2 task, we asked the participants to mentally rotate the objects on the right in each pair at the speed with which the objects had rotated when they viewed them (i.e., during the VR task).

In the mental rotation tasks, the procedure was identical to the one used in the SM task of Experiment 1. Each trial started with a $250 \mathrm{~ms}$ blank screen, then one of the stimuli was presented until participants responded by pressing one of two buttons, within a $7500 \mathrm{~ms}$ time limit. We asked participants to decide whether or not the two objects had the identical shape by mentally rotating the object on the right into congruence with the object on the left. Participants used their dominant hand to respond, using the same response keys ("b" and " $n$ ") as in Experiment 1. The onset of a stimulus started a timer, which was stopped when either of the two response keys was pressed.

In each mental rotation task (MR1 and MR2), participants performed two blocks of 48 trials, for a total of 96 trials per task. In each block, six objects were presented eight times, with the four orientations appearing equally often. On half of the trials the objects were the same shape; on half, they were mirror images. An object used in one block was never used in any of the other blocks. The four sets of objects in each of the blocks of the two tasks were fully counterbalanced over participants in each group. The order of the trials was random, except that no 
more than three same or different trials occurred consecutively and the same object could not appear consecutively. In each mental rotation task, participants performed 12 practice trials, where the computer provided feedback on their answers, prior to the experimental trials.

In the visual rotation task, after a $250 \mathrm{~ms}$ blank screen, an animation of one of the 12 objects was presented in the center of the screen. Participants performed two blocks of 48 trials for a total of 96 trials. On half of the trials, objects rotated clockwise; on half, they rotated counterclockwise. Trials were presented randomly except that no more than three rotations of the same direction and of the same object occurred consecutively. In the fast condition of the VR task, objects took $2 \mathrm{~s}$ to complete a full rotation (360 degrees), at a rotation speed of $5.5 \mathrm{~ms} /$ degree. In the slow condition of the VR task, objects took $8 \mathrm{~s}$ to complete a full rotation, at a rotation speed of $22.2 \mathrm{~ms} /$ degree. After the object had fully rotated, the participants were to use their dominant hand to press the "b" key when the object had rotated clockwise or the " $\mathrm{n}$ " key when it had rotated counterclockwise. We asked the participants to delay their response until the object had finished its 360-degree rotation, which thus required the participants to watch the display. The response and the time taken to respond were recorded.

At the end of the experiment, the participants completed the same paperand-pencil questionnaires as in Experiment 1. 


\section{Results}

As in Experiment 1, analyses of RTs in the mental rotation tasks included only data from trials where the two shapes matched and on which participants provided the correct answer. Outliers, defined as in Experiment 1, occurred on $1.9 \%$ of the trials. After removing the outliers for each participant, the average RT for each angle of rotation in each of the two mental rotation tasks was computed.

In the VR task, we limited our analysis to the accuracy of participants' responses, in order to verify that participants did in fact pay attention to the objects' rotations. In both conditions of the VR task, the participants were extremely accurate when judging the direction of rotation (with fewer than $1 \%$ errors). Thus, we are confident that participants focused their attention on the rotations of the novel objects. No further analyses of the VR task are provided in the subsequent analysis of the results.

Our goal was to discover whether the rotation speed of an object presented perceptually affects the slope of the best-fitting lines in a mental rotation task. A prerequisite for this analysis is to ensure that mental rotation was used in the mental rotation tasks in each of the two groups. Thus, as a first step, we analyzed the mental rotation data from each of the two groups to determine whether RTs increased linearly with increasing angle. Following this, we conducted a systematic comparison of the ERs, the steepness of the slopes of the best-fitting 
lines, and the heights of the RT intercepts for the MR1 task versus the MR2 task for each of the two groups.

We observed no effect of gender on any of the dependent variables. Thus, we pooled the data over this variable. Finally, none of the scores on the three questionnaires differed between participants assigned either to the fast or the slow VR groups.

\section{Effect of angle}

Slow VR task group. In both mental rotation tasks, the angular disparity between the two objects affected RTs, as revealed by one-way repeated ANOVAs, with $F(3,33)=57.55, p<.0001, \eta p^{2}=.84$ for the MR1 task and $F(3$, $33)=47.93, p<.0001, \eta p^{2}=.82$, for the MR2 task. As is evident in Figure 5, RTs increased linearly with the difference in angles between the two objects in the MR1 $\left[F(1,11)=163.01, p<.0001, \eta p^{2}=.83\right]$ and the MR2 tasks $[F(1,11)=$ 74.86, $\left.\mathrm{p}<.0001, \eta \mathrm{p}^{2}=.81\right]$. The quadratic components of the angular disparity effect were significant in the MR1 task, $F(1,11)=9.13, p<.025, \eta p^{2}=.22$, and in the MR2 task, $\mathrm{F}(1,11)=6.96, \mathrm{p}<.025, \eta \mathrm{p}^{2}=.17$. However, the proportion of variance explained by the linear trend was, respectively, $94.9 \%$ in the MR1 task and $95 \%$ in the MR2 task. Finally, replicating other studies of mental rotation, RTs were highly correlated with the magnitude of the difference in orientation of the two objects, with rs $=.97, \mathrm{ps}<.05$ in both tasks (see Figure 5). 
Insert Figure 5 about here

Fast VR task group. In both mental rotation sessions, the angular disparity between the two objects affected RTs, as revealed by one-way repeated ANOVAs, with $\mathrm{F}(3,33)=50.42, \mathrm{p}<.0001, \eta \mathrm{p}^{2}=.82$, for the MR1 task and $\mathrm{F}(3$, $33)=40.78, p<.0001, \eta p^{2}=.79$, for the MR2 task. As reported for the slow VR group, RTs increased linearly with increasing angles in the first set of MR trials, F $(1,11)=141.62, \mathrm{p}<.0001, \eta p^{2}=.81$, and in the second set of MR trials, $\mathrm{F}(1,11)$ $=121.46, \mathrm{p}<.0001, \eta \mathrm{p}^{2}=.78$. The trend analysis revealed a significant quadratic component in the MR1 task, $\mathrm{F}(1,11)=8.26, \mathrm{p}<.025, \eta \mathrm{p}^{2}=.20$ but not in the MR2 task, $\mathrm{F}<1, \eta \mathrm{p}^{2}=.03$. In addition, the linear trend accounted for $93.6 \%$ of the variance of the orientation effect in the first set of trials and $99.3 \%$ of the variance in the second set of trials. As shown in Figure 6, RTs were strongly correlated with angular disparity in both the MR1 task, $\mathrm{r}(2)=.97, \mathrm{p}<.05$, and the MR2 task, $\mathrm{r}(2)=.99, \mathrm{p}<.01$.

Insert Figure 6 about here 
ERs, slopes and intercepts

ERs. The ERs did not differ significantly between the MR1 task and the MR2 task, either in the slow VR task group, $\mathrm{M}=18.4 \%$ versus $\mathrm{M}=16.8 \%, \mathrm{t}(11)$ $<1, d=.17$, or in the fast VR group, $\mathrm{M}=15.8 \%$ versus $\mathrm{M}=17 \%, \mathrm{t}<1, d=.11$ (see Table 2). In addition, a two-way ANOVA (MR task x VR groups) did not reveal an interaction between the type of VR task (i.e., slow versus fast) and the type of MR task (i.e., MR1 and MR2), $F<1, \eta p^{2}=.03$.

Insert Table 2 about here

Slopes. We again failed to observe an interaction between the VR condition and the MR tasks on the RTs, F $(1,22)=1.34, p>.25, \eta p^{2}=.06$. As in Experiment 1, we conducted a power analysis of the interaction between the VR conditions (i.e., slow vs. fast) and the MR tasks (i.e., MR1vs. MR2 tasks) on the slopes to estimate the risk of a type-II error. According to the tacit knowledge account, we would expect at least a moderate effect size for the interaction (e.g. $\eta^{2}$ $=.10$, which is on the small side for an effect size). However, for $\alpha=.05$, a sample of 24 participants, and a correlation of $\mathrm{r}(22)=.43$ between the slopes in the MR1 and MR2 tasks, the probability of a type-II error is low $(\beta=4.7 \%)$. In addition, the mental rotation rates were comparable before $(\mathrm{M}=10.7 \mathrm{~ms} /$ degree 
in the slow VR task group and $\mathrm{M}=11.2 \mathrm{~ms} /$ degree in the fast VR task group) and after $(\mathrm{M}=11.4 \mathrm{~ms} /$ degree in the slow $\mathrm{VR}$ task group and $\mathrm{M}=9.8 \mathrm{~ms} /$ degree in the fast VR task group) the visual rotation task, all ts $<1, d s<.24$ (see Table 2).

Moreover, in the slow VR task group, the rotation speed of the objects presented visually ( $22.2 \mathrm{~ms} /$ degree) was not within the $99 \%$ CI of the mental rotation rate observed in the MR2 task $(\mathrm{CI}=8.1 \mathrm{~ms} /$ degree $-14.8 \mathrm{~ms} /$ degree $)$. Similarly, in the fast VR task group, the rotation rate of the objects in the VR task (5.5 ms/degree) was outside the $99 \%$ CI of the speed of mental rotation in the MR2 task $(\mathrm{CI}=6.7 \mathrm{~ms} /$ degree $-12.8 \mathrm{~ms} /$ degree $)$.

Finally, we compared the speed of mental rotation between the fast and the slow VR groups. We conducted an analysis of covariance (ANCOVA) on the mental rotation rates in the MR2 task, with the rotation rates in the MR1 task as a covariate and the type of VR task as a fixed factor. As expected, there was no difference between the two groups in the speed of rotation in the second set of trials, $\mathrm{F}(1,21)=1.24, \mathrm{p}>.25, \eta \mathrm{p}^{2}=.06$.

Intercepts. As in the two previous experiments, we considered the average RT in the 0 degree orientation as the height of the intercept (see Table 2). In the slow VR task group, the intercept was no higher in the MR2 task $(M=1767 \mathrm{~ms})$ than in the MR1 task $(\mathrm{M}=1390 \mathrm{~ms}), \mathrm{t}(11)=1.61, \mathrm{p}>.10, d=.51$. Similarly, in the fast VR task group, the heights of the intercepts did not differ between the 
MR1 task $(\mathrm{M}=1735 \mathrm{~ms})$ and the $\mathrm{MR} 2$ task $(\mathrm{M}=1387 \mathrm{~ms}), \mathrm{t}(11)=1.05, \mathrm{p}>.25$, $d=.39$.

However, crucially, we did find evidence that the instructions were not simply ignored: We found an interaction on the intercepts between the VR group and the MR task, $F(1,22)=4.48, p<.05, \eta p^{2}=.27$. As shown in Figure 7 , participants slowed down their responses after having seen the relatively slow perceptual display in the slow VR condition, and sped up their responses after having seen the relatively fast perceptual display in the VR condition. This interaction suggested that participants regulated their responses in the second set of MR trials in an attempt to mimic what they had seen.

Insert Figure 7 about here

\section{Discussion}

Even though participants were explicitly instructed to mentally rotate objects at the same rate that they saw objects rotating in the visual rotation task, the steepness of the slopes was comparable in the mental rotation task performed before and after the visual rotation task for both groups of participants. A lack of statistical power cannot account for our failure to find differences in the speed of mental rotation after observing rotating three-dimensional shapes. First, the 
difference in the steepness of the slopes did not exceed .25 standard deviations. Second, the probability of a type-II error in the analysis of the interaction between Task and VR groups on the slopes was less than 5\%. Third, the lack of difference on the slopes replicated the results of Experiment 1. Finally, there was no difference in the mental rotation speed between the VR slow group and the VR fast group in the MR2 task, when controlling for the speed of the rotation in the MR1 task -- as revealed by an ANCOVA, which increases the power of the analysis.

However, we did find that the intercept of these slopes was different for the two groups: For participants who had viewed quickly rotating objects, the height of the intercepts was lower in the MR2 task than it was for participants who viewed the slowly rotating objects. Thus, we are confident that participants did attempt to modify the speed of their responses.

Although participants succeeded in delaying (in the slow VR task group) or speeding up (in the fast VR task group) their overall responses, participants were not able to alter the speed of rotation per se (as reflected in the slopes of the best-fitting lines). The speed with which participants mentally rotated the objects in their images was not affected by their knowledge of the world (i.e., the rotation speed of the visually presented objects); the participants were not able to use this knowledge to mimic what would happen when they imagined the corresponding situation (i.e., during the mental rotation task). Nevertheless, the effect on the 
intercept is important in part because it demonstrates that we had enough power to detect effects. Moreover, it shows that our participants were in fact trying to cooperate. Finally, it shows that the overall speed of responding can be altered at will, but the perhaps more complex mental rotation processes are not easily modified. Overall, these results suggest that the rotational component of mental rotation cannot be easily modified or affected by task demands.

\section{General Discussion}

As suggested by Pylyshyn (1981, 2003, 2007), we treated tacit knowledge as the "null hypothesis" to explain the mental rotation effect. In Experiment 1, we used realistic sets of instructions to change participants' knowledge and expectations. In Experiment 2, we presented objects visually rotating at different speeds to modify participants' beliefs and knowledge of the way our novel stimuli behave in the real world. In no case did such knowledge affect the rate of mental rotation.

These results taken together allow us to argue that, in general, tacit knowledge and cognitive penetration are not pervasive factors that affect mental rotation experiments. And, in particular, we showed that these factors do not provide the best explanation of the linear relationship between RTs and angular disparity that is consistently reported in the mental rotation literature (e.g., see Shepard \& Cooper, 1982). Instead, the linear increase in RT with increasing 
angular disparity suggests that intermediate representations of the object need to be visualized in order to mentally rotate an object. One cannot easily imagine an object in one orientation simply being transposed to another orientation as opposed to what has been reported for image scanning processes (e.g., Kosslyn, 1980).

The results of Experiment 2 demonstrated that even the speed of mental rotation is not easily cognitively penetrable. Of course, we cannot rule out that the speed of rotation conceivably could be altered in some circumstances, but we have shown that it is not easily modified - as it should be if tacit knowledge were governing performance.

In short, the findings in the two experiments reported in this article suggest that the tacit knowledge account is not a plausible explanation for all imagery effects. Instead, at least some such effects apparently are constrained by the intrinsic nature of the underlying representations and processes used to perform the task. In particular, the remarkable consistency of the rotation rate suggests that the speed of rotation is constrained by characteristics of the underlying representations and processes that are used in mental rotation. 


\section{References}

Blajenkova, O., Kozhevnikov, M., \& Motes, M. A. (2006). Object-Spatial imagery: A new self-report imagery questionnaire. Applied Cognitive Psychology, 20, 239-263.

Carpenter, M. A., \& Just, P. A. (1985). Cognitive coordinate systems: Accounts of mental rotation and individual differences in spatial ability. Psychological Review, 92, 137-172.

Carpenter, P. A., \& Just, M. A. (1978). Eye fixations during mental rotation. In J. W. Senders, D. F. Fisher, \&. R. A. Monty (Eds.), Eye movements and the higher psychological functions (pp. 115-133). Hillsdale, NJ: Erlbaum.

Cohen, J. (1988). Statistical power analysis for the behavioral sciences (2nd Ed.). Hillsdale, NJ: Lawrence Erlbaum Associates.

Cooper, L. A. (1976). Individual differences in visual comparison processes. Perception \& Psychophysics, 19, 433-444.

Cooper, L. A., \& Shepard, R. N. (1973). Chronometric studies of the rotation of mental images. In W. G. Chase (Eds.), Visual information processing (pp. 75-176). New York: Academic Press.

Faul, F., Erdfelder, E., Lang, A.-G., \& Buchner, A. (2007). G*Power 3: A flexible statistical power analysis program for the social, behavioral, and biomedical sciences. Behavior Research Methods, 39, 175-191. 
Geiser, C., Lehmann, W., and Eid, M. (2006). Separating "rotators" from "nonrotators" in the mental rotation test: A multigroup latent class analysis. Multivariate Behavioral Research, 41, 261-293.

Jolicoeur, P. (1985). The time to name disoriented natural objects. Memory and Cognition, 13, 289-303.

Just, P. A., \& Carpenter, M. A. (1976). Eye fixations and cognitive processes. Cognitive Psychology, 8, 441-480.

Koriat, A., \& Norman, J., (1985). Reading rotated words. Journal of Experimental Psychology: Human Perception and Performance, 11, 490-508.

Kosslyn, S. M. (1980). Image and mind. Cambridge, MA: Harvard University Press.

Kosslyn, S. M., Ball, T. M., \& Reiser, B. J. (1978). Visual images preserve metric spatial information: Evidence from studies of image scanning. Journal of Experimental Psychology: Human Perception and Performance, 4, 47-60.

Kosslyn, S. M., Thompson, W. L., \& Ganis, G. (2006). The case for mental imagery. New York: Oxford University Press.

Marks, D. F. (1973). Visual imagery differences in the recall of pictures. British Journal of Psychology, 64, 17-24.

Oldfield, R. C. (1971). The analysis and assessment of handedness: The Edinburgh inventory. Neuropsychologia, 9, 97-113. 
Pylyshyn, Z. W. (1979). The rate of "mental rotation" of images: A test of a holistic analogue hypothesis. Memory and Cognition, 7, 19-28.

Pylyshyn, Z. W. (1981). The imagery debate: Analogue media versus tacit knowledge. Psychological Review, 87, 16-45.

Pylyshyn, Z. W. (2002). Mental imagery: In search of a theory. Behavioral and Brain Sciences, 25, 157-238.

Pylyshyn, Z. (2003). Return of the mental image: Are there pictures in the brain? Trends in Cognitive Sciences, 7, 113-118.

Pylyshyn, Z. W. (2003). Seeing and Visualizing: It's not what you think. Cambridge, MA: MIT Press.

Shepard, R. N., \& Cooper, L. A. (1982). Mental images and their transformations. Cambridge, MA: The MIT Press.

Shepard, R. N., \& Metzler, J. (1971). Mental rotation of three-dimensional objects. Science, 171, 701-703.

Shepard, S., \& Metzler, D. (1988). Mental rotation: Effect of dimensionality of objects and type of task. Journal of Experimental Psychology: Human Perception and Performance, 14, 3-11.

Tarr, M. J., \& Pinker, S. (1989). Mental rotation and orientation-dependence in shape recognition. Cognitive Psychology, 21, 233-282. 
Thompson, W. L., Kosslyn, S. M., Hoffman, M. S., and van der Kooij, K. (2008). Inspecting visual mental images: Can people "see" implicit properties as easily in imagery and perception? Memory and Cognition, 26, 1024-1032.

Vandenberg, S. G., \& Kuse, A. R. (1978). Mental rotation, a group test of threedimensional spatial visualization. Perceptual and Motor Skills, 60, 343-350.

Wright, R., Thompson, W. L., Ganis, G., Newcombe, N. S., \& Kosslyn, S. M. (2008). Training generalized spatial skills. Psychonomic Bulletin \& Review, 15, 763-771. 


\section{Appendix}

Mental rotation instructions:

"We have found that the most efficient way to perform this task is the following: When you see the two objects, please mentally rotate (in your mind's eye) the object on the right from its original position to a position where it is aligned in the same way as the object on the left. Once the objects are in alignment, that is, once they are oriented in the same way, please make your judgment as to whether they are the same or different. It is important that, on each trial, you "see" the object on the right side of the screen rotating from its position on the screen into alignment with the object on the left side."

Leap instructions:

"We have found that the most efficient way to perform this task is the following: When you see the two objects, please imagine (in your mind's eye) that the object on the right quickly and in a single leap, jumps from its original position to a position where it is aligned in the same way as the object on the left. Once the objects are in alignment, that is, once they are oriented in the same way, please make your judgment as to whether they are the same or different. It is important that, on each trial, you "see" the object on the right side of the screen jumping quickly and in a single leap, without visualizing the intermediate steps 
(as if the transformation was instantaneous), from its position on the screen into alignment with the object on the left side."

Analytic instructions:

"We have found that the most efficient way to perform this task is by using an analytical approach. That is, in order to accomplish this task most quickly and accurately, it will be easiest if you compare the segments of the object on the right with the segments of the object on the left, to decide whether they fit together in the same way without rotating any of the segments." 
Mental Rotation and Cognitive Penetration, p.44

\section{Acknowledgements}

This research was supported by the National Institutes of Health (NIH), under Grant R01 MH060734; any opinions, findings, and conclusions or recommendations expressed in this material are those of the authors and do not necessarily reflect the views of NIH. We are grateful to Jacob Kantrowitz-Sirotkin and Katie Lewis for their help in recruiting participants and collecting data. 
Mental Rotation and Cognitive Penetration, p.45

Table 1. Experiment 1: Mean RTs for different orientations (ms), mean slopes of the best-fitting lines (ms/degree), mean ERs (\%) and standard errors of the mean (SE) in the SM task and the CS task for different types of instructions.

\begin{tabular}{|c|c|c|c|c|c|c|c|c|c|c|c|c|c|c|}
\hline & & & \multicolumn{12}{|c|}{ Type of tasks } \\
\hline & & & \multicolumn{6}{|c|}{ SM task } & \multicolumn{6}{|c|}{ CS task } \\
\hline & & & \multicolumn{4}{|c|}{ Angular disparity between objects (degree) } & \multirow[b]{2}{*}{ Slopes } & \multirow[b]{2}{*}{ ERs } & \multicolumn{4}{|c|}{ Orientation of the stimuli (degree) } & \multirow[b]{2}{*}{ Slopes } & \multirow[b]{2}{*}{ ERs } \\
\hline & & & 0 & 50 & 100 & 150 & & & 0 & 60 & 120 & 180 & & \\
\hline \multirow{6}{*}{$\begin{array}{c}\text { Type of } \\
\text { instruction }\end{array}$} & \multirow[b]{4}{*}{ Leap } & $\mathrm{M}$ & 1516 & 2364 & 3098 & 3329 & 12.4 & 12.5 & 822 & 946 & 1122 & 1583 & 4.1 & 5.2 \\
\hline & & SE & 128.19 & 165.63 & 186.86 & 182.25 & 1.17 & 2.27 & 31.58 & 39.42 & 41.49 & 94.30 & 0.48 & 0.89 \\
\hline & & $\mathrm{M}$ & 1407 & 2264 & 2816 & 3141 & 11.5 & 13.1 & 814 & 911 & 1043 & 1426 & 3.3 & 4.4 \\
\hline & & SE & 84.95 & 167.99 & 193.29 & 194.96 & 1.10 & 1.88 & 39.43 & 44.44 & 46.98 & 97.05 & 0.42 & 1.32 \\
\hline & \multirow[b]{2}{*}{ Analytic } & $\mathrm{M}$ & 1395 & 2323 & 2961 & 3315 & 12.8 & 11.8 & 924 & 1006 & 1235 & 1682 & 4.2 & 4.8 \\
\hline & & SE & 61.63 & 143.99 & 189.61 & 216.22 & 1.18 & 1.52 & 53.87 & 37.47 & 77.35 & 98.67 & 0.45 & 1.21 \\
\hline
\end{tabular}


Table 2. Experiment 2: Mean RTs for different orientations (ms), mean slopes of the best-fitting lines (ms/degree), mean ERs (\%) and standard errors of the mean (SE) in the MR1 and the MR2 tasks for different types of VR task.

\begin{tabular}{|c|c|c|c|c|c|c|c|c|c|c|c|c|c|c|}
\hline & & & \multicolumn{12}{|c|}{ Type of tasks } \\
\hline & & & \multicolumn{6}{|c|}{ MR1 task } & \multicolumn{6}{|c|}{ MR2 task } \\
\hline & & & \multicolumn{4}{|c|}{ Angular disparity between objects (degree) } & \multirow[b]{2}{*}{ Slopes } & \multirow[b]{2}{*}{ ERs } & \multicolumn{4}{|c|}{ Angular disparity between objects (degree) } & \multirow[b]{2}{*}{ Slopes } & \multirow[b]{2}{*}{ ERs } \\
\hline & & & 0 & 50 & 100 & 150 & & & 0 & 50 & 100 & 150 & & \\
\hline \multirow{4}{*}{$\begin{array}{c}\text { Type } \\
\text { of VR } \\
\text { task }\end{array}$} & \multirow[b]{2}{*}{ Slow } & $\mathrm{M}$ & 1390 & 2145 & 2774 & 2963 & 10.7 & 18.4 & 1767 & 2573 & 3222 & 3452 & 11.4 & 16.8 \\
\hline & & SE & 128.61 & 203.55 & 229.07 & 251.29 & 1.10 & 3.02 & 267.92 & 283.09 & 276.17 & 283.99 & 1.32 & 3.74 \\
\hline & \multirow[b]{2}{*}{ Fast } & $\mathrm{M}$ & 1666 & 2420 & 3143 & 3292 & 11.2 & 15.8 & 1497 & 2077 & 2564 & 2960 & 9.8 & 17.0 \\
\hline & & SE & 188.79 & 270.88 & 278.73 & 282.16 & 1.09 & 2.84 & 137.20 & 174.00 & 186.93 & 192.08 & 1.18 & 2.65 \\
\hline
\end{tabular}




\section{Figure Captions}

Figure 1. Example of a pair of three-dimensional objects with (a) identical and (b) different shapes with a $50^{\circ}$ rotation of the object on the right used in the SM task.

Figure 2. Example of a (a) standard and a (b) mirror-image character rotated by $120^{\circ}$, used in the CS task.

Figure 3. Experiment 1. RTs for different angular disparities in the threedimensional shapes for the three groups of participants in the SM task.

Figure 4. Experiment 1. RTs for different angular disparities of the character from its upright orientation for the three groups of participants in the CS task.

Figure 5. Experiment 2. RTs for different angular disparities for the two mental rotation tasks (i.e., MR1 and MR2) for the slow VR task group.

Figure 6. Experiment 2. RTs for different angular disparities for the two mental rotation tasks (i.e., MR1 and MR2) for the fast VR task group.

Figure 7. Experiment 2. RTs for the intercepts for the two mental rotation tasks (MR1 and MR2) for the fast and slow VR task groups. Error bars represent standard errors of the mean. 


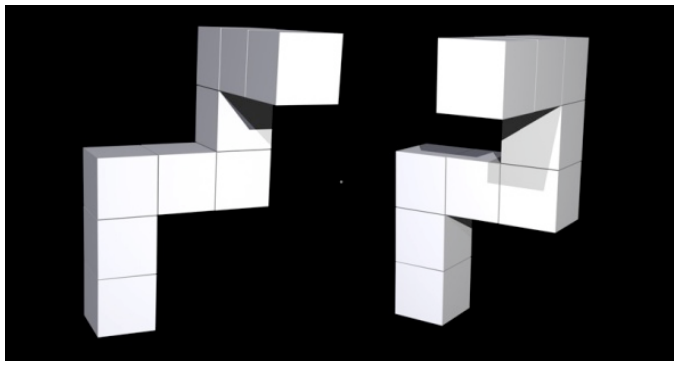

(a)

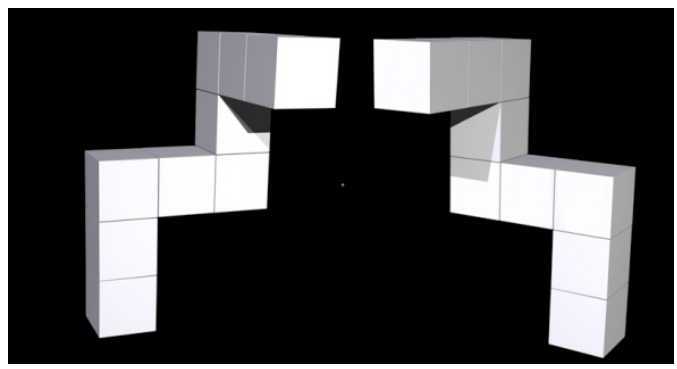

(b)

Figure 1. Example of a pair of three-dimensional objects with (a) identical and (b) different shapes with a $50^{\circ}$ rotation of the object on the right used in the SM task. 
Mental Rotation and Cognitive Penetration, p.49

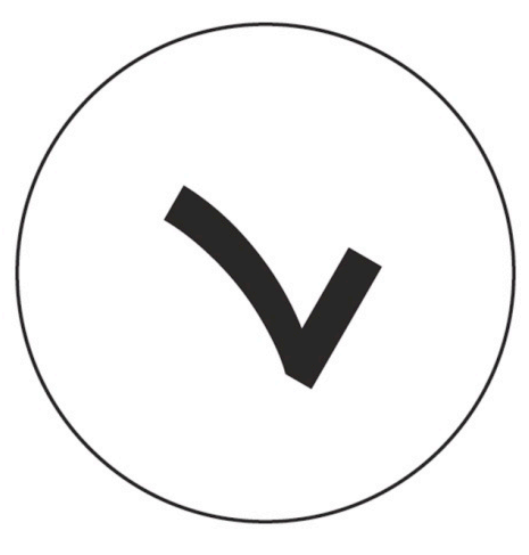

(a)

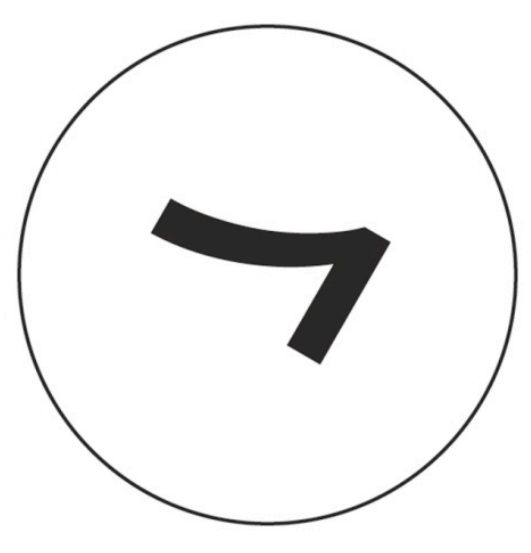

(b)

Figure 2. Example of a (a) standard and a (b) mirror-image character rotated by $120^{\circ}$, used in the CS task. 


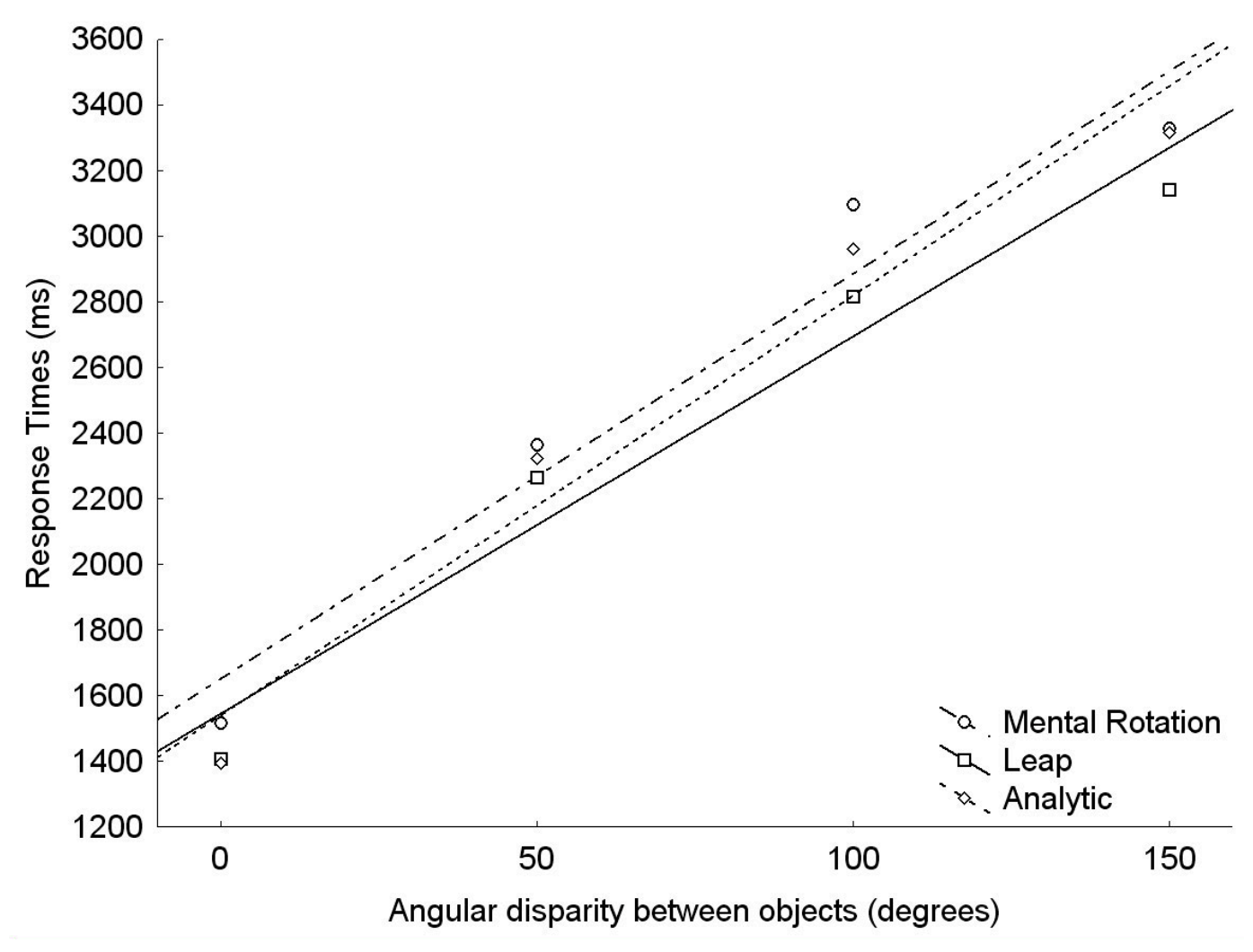

Figure 3. Experiment 1. RTs for different angular disparities in the threedimensional shapes for the three groups of participants in the SM task. 


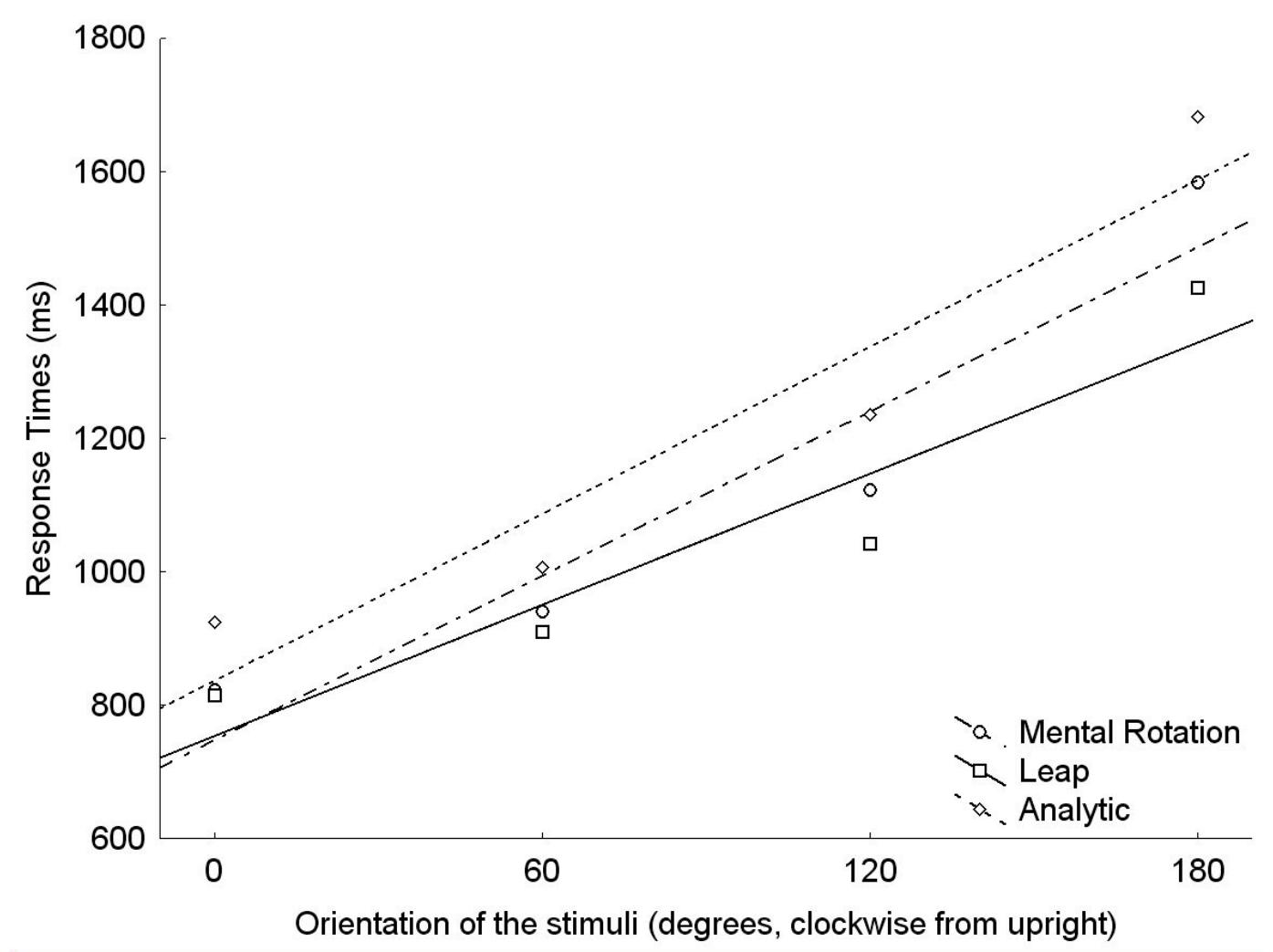

Figure 4. Experiment 1. RTs for different angular disparities of the character from its upright orientation for the three groups of participants in the CS task. 


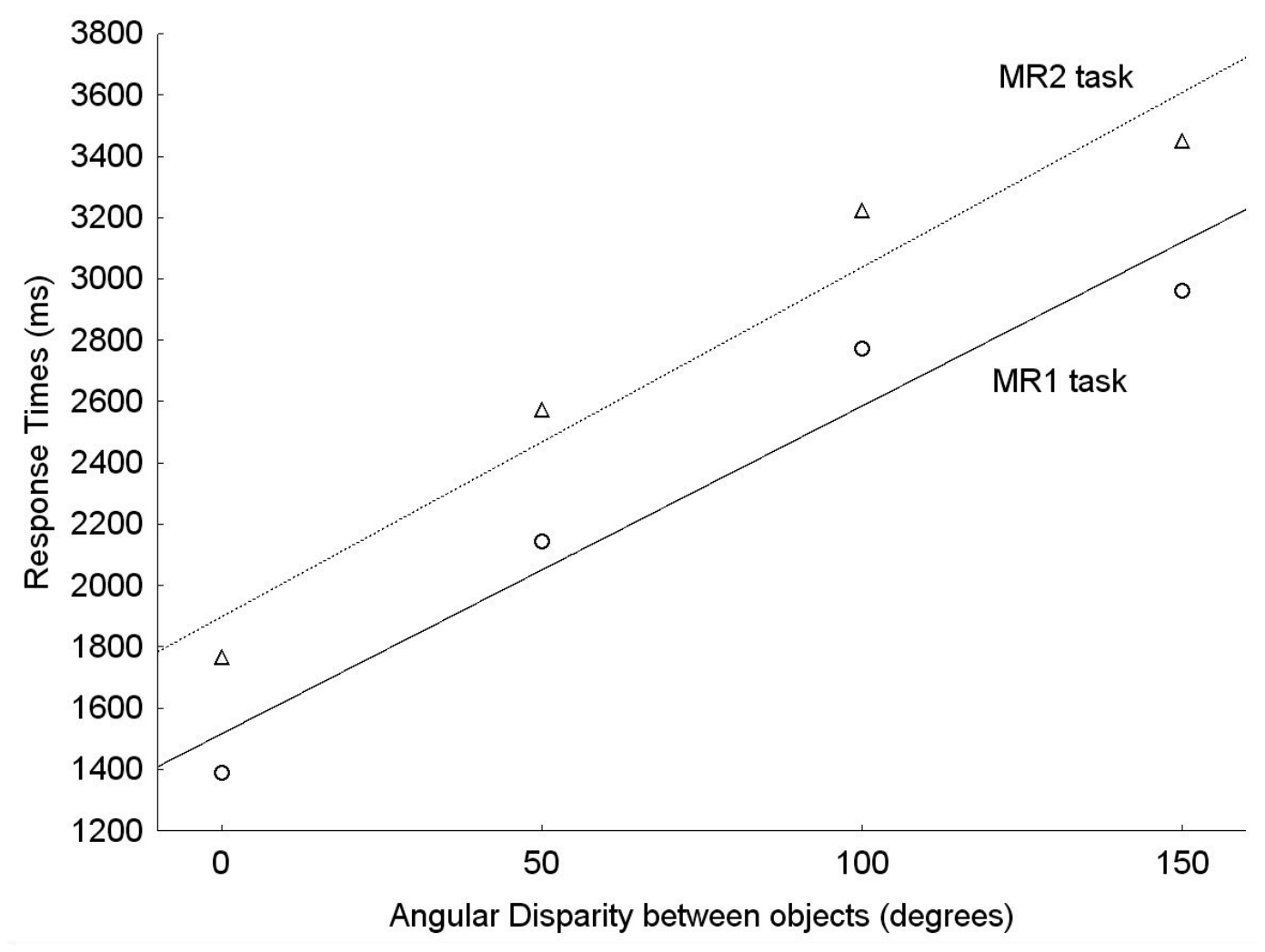

Figure 5. Experiment 2. RTs for different angular disparities for the two mental rotation tasks (i.e., MR1 and MR2) for the slow VR task group. 


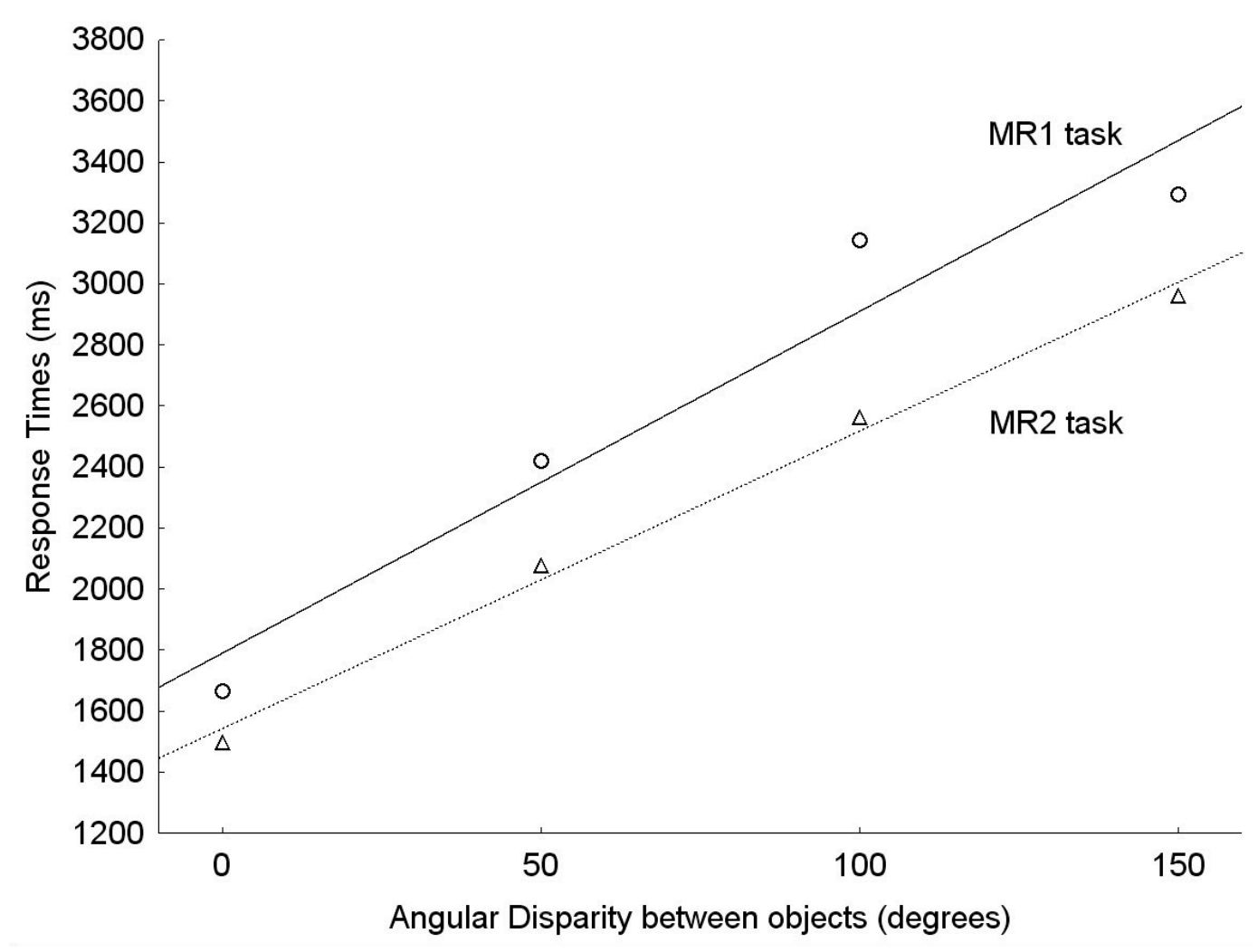

Figure 6. Experiment 2. RTs for different angular disparities for the two mental rotation tasks (i.e., MR1 and MR2) for the fast VR task group. 


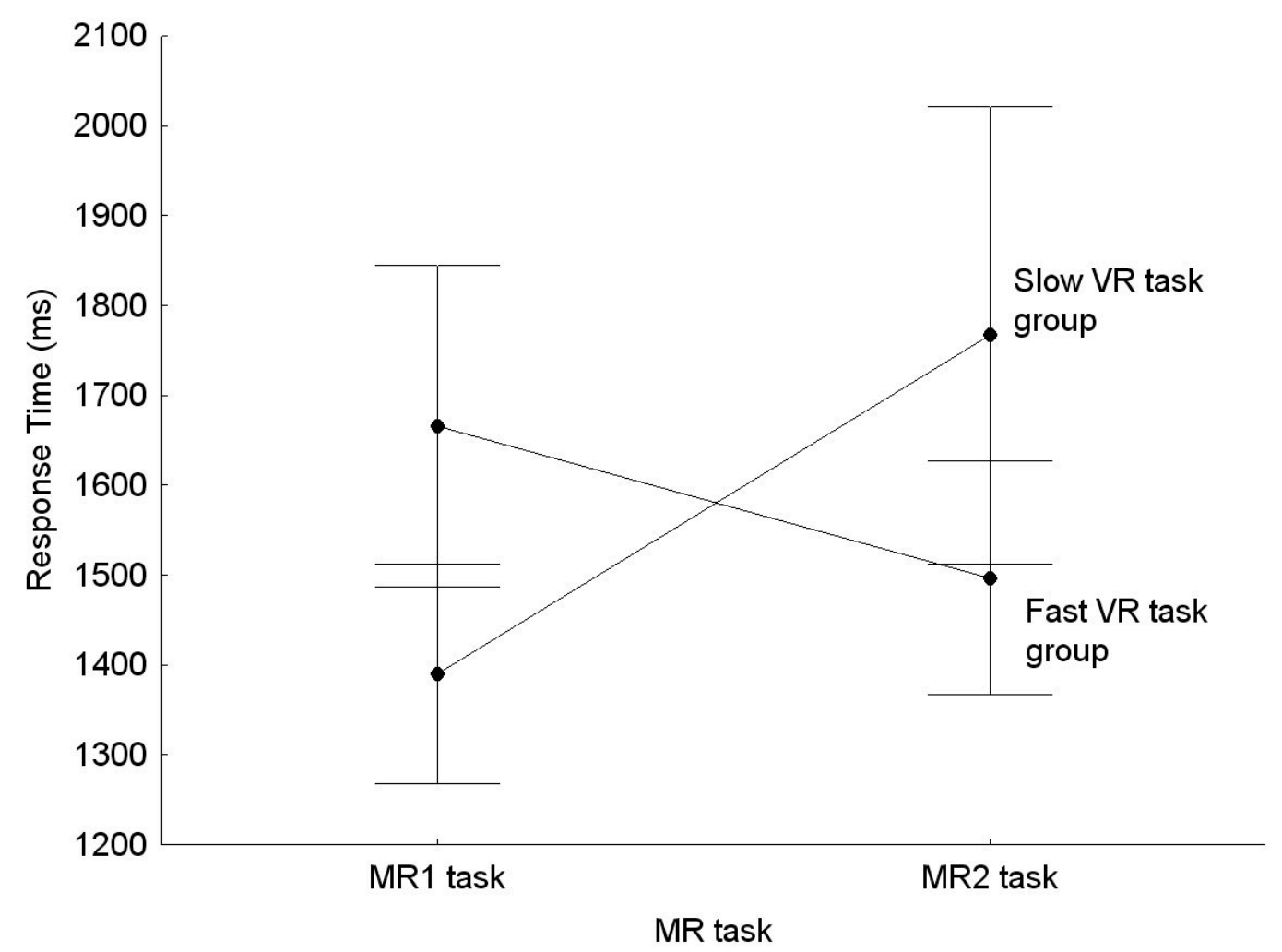

Figure 7. Experiment 2. RTs for the intercepts for the two mental rotation tasks (MR1 and MR2) for the fast and slow VR task groups. Error bars represent standard errors of the mean. 